\title{
An application of statistical modelling on power generating asset performance analysis at the Asam-asam steam power plant of South Kalimantan
}

\author{
Aqli Mursadin ${ }^{1, *}$ \\ ${ }^{1}$ Faculty of Engineering, Lambung Mangkurat University, Banjarmasin 70123
}

\begin{abstract}
A power generating system in a steam power plant is a complex one. This system involves a large number of variables containing information concerning the operation conditions. It can also be seen as an important asset in both power generation and energy portfolios. At the Asam-asam power plant of South Kalimantan, the complexity of the system has led to difficulties in explaining the apparently steady decrease in the output power. The amount of data collected is simply too big and the dimension too high for analysis purposes based on conventional thermodynamics. This study was performed to tackle the problem using statistical modelling. This approach can accommodate empirical behaviors of the variables and the probabilistic nature of the system. Information obtained from this modelling can be valuable for various purposes. The method consists of literature review, model development, data collection and analysis, and model fitting. Generalized additive models were chosen. Data were available from the company as observed from more than 140 variables. The resulting model identifies variables significantly related to the output power and locate subsystems whose fluctuating behaviors are usually ignored in a conventional thermodynamic analysis. A direction for future research is recommended.
\end{abstract}

\section{Introduction}

A power generating system in a steam power plant is a complex one. It converts chemical energy in the fuel supplied to the system into heat. The circulating water fed to the boiler as liquid captures this heat. This surge in energy is such that it turns the liquid water into superheated vapor, which then turns around the blades of the turbine. The resulting mechanical work becomes electricity through the coupling of the rotor with the shaft of an electrical generator. This complex mechanism works in a cycle within a configuration of equipment [1, 2].

Such a complex system involves a large number of variables. These variables carry with them information concerning the operation conditions of the plant. Consequently, analysis of these variables and the relationships between them is essential for explaining the behavior and the performance of the system. These variables can be considered as random variables whose observed values are subject to random chances, and their analysis requires an approach that accommodates their probabilistic behaviors.

Further, such a system can be seen as an important asset in both power generation and energy asset portfolios. The performance of this system requires the use of statistical techniques as pointed out by [3] in the context of equipment and process monitoring in engineering asset management. A promising approach is based on modelling such behaviors.

The Asam-asam steam power plant has four separate generating units located in the Asam-asam Village of the Tanah Laut Regency $122 \mathrm{~km}$ northeast of Banjarmasin. Of these generating units, Unit 1 was initially synchronized into the grid on 28 June 2000, Unit 2 on 25 October 2000, Unit 3 on 24 March 2012, and Unit 4 on 7 November 2012. Each of these units was originally designed to have a maximum output power of $65 \mathrm{MW}$.

Data collected until December 2016 indicate that there has been a steady decrease in the output of Unit 2 with the lowest value of around 85 percent of the maximum. A study by [4] shows that the vacuum pressure at the main steam condenser has an increase of 20 percent. This may suggest that the main steam leaving the turbine still has a sufficiently high enthalpy (total energy), or the fouling growth inside the condenser is unusually rapid, or else. Any one of these may contribute to the above-mentioned decrease in the output. However, this study only considered variables observed on equipment and systems directly connected to the turbine. For examples, several devices like the deaerator (used to remove gas from liquid feed water) and the pulverizer (which supplies the coal powder) may perform processes

Corresponding author: a.mursadin@gmail.com 
whose variables fluctuate in values in such a pattern consistent with that of output power.

It is difficult to explain this steady decrease due to the high dimension of variables and the big size of data. It is also worsen by the fact that the available measurement data have never been properly used for studying the system.

An ability to model the relationships between these process variables can be useful in uncovering information concerning the operation behaviors of the system. This, in turn, can provide knowledge and understanding beneficial to the maintenance of the power plant and the improvement of its performance in the future.

For this reason, it is important to study how it is possible to model the power generating behavior of the system in such a way that completely explains the effect of each of the system variables. This study should give insight into the empirical behavior of the system. It can provide a set of models with which the relationship between system variables can be represented and analyzed. The resulting information will be useful for operation and maintenance purposes as well as for future improvement of the system.

\section{A problem of decreasing output power}

A steam power plant utilizes total energy or enthalpy of circulating water to generate electricity. It basically consists of several main components including a steam turbine, a boiler, a heat exchanger known as a condenser, a water feed pump that supplies condensed water to the boiler, and a complex piping system along with several other auxiliary devices and pieces of equipment. The rotating shaft of the turbine is then coupled with its counterpart in the electrical generator to produce electricity. This generating system works in a cycle. A theoretical introduction to this type of power plant from the thermodynamic viewpoint can be found in [1].

A modern modification of the above cycle is the one currently installed in the Asam-asam power plant, especially the generating unit 2. Here, several other devices are added such as a low-pressure heater, a highpressure heater, a deaerator, and an economizer. Fig. 1 shows the diagram of the cycle at this generating unit.

Processes in such a cycle usually involve a large number of variables. For instance, at the generating unit 2 as mentioned above, more than 140 variables are monitored and their values logged every two hours on a daily basis.

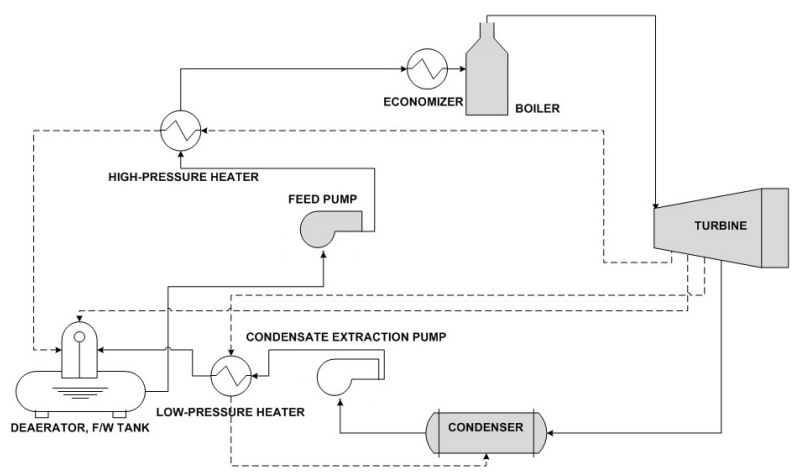

Fig. 1. A diagram of the generating cycle at the generating unit 2 of Asam-asam.

As previously mentioned, there has been a problem of decreasing output power at the Asam-asam power plant. The worst case can be found at the generating unit 2 where the lowest output power is around 85 percent of its maximum. Fig. 2 shows the empirical distribution of the output power at the generating unit 2 of the plant during the period between 01/12/2016 and 14/12/2016 (in MW). Apparently, the average output is far below its maximum capacity.

Moreover, the above mentioned period saw several processes involved in the generating system of that particular unit being in their unstable conditions. This means that the data simply represent those processes' states of instability. This is, however, a short period before a general overhaul was carried out in the beginning of the following year.

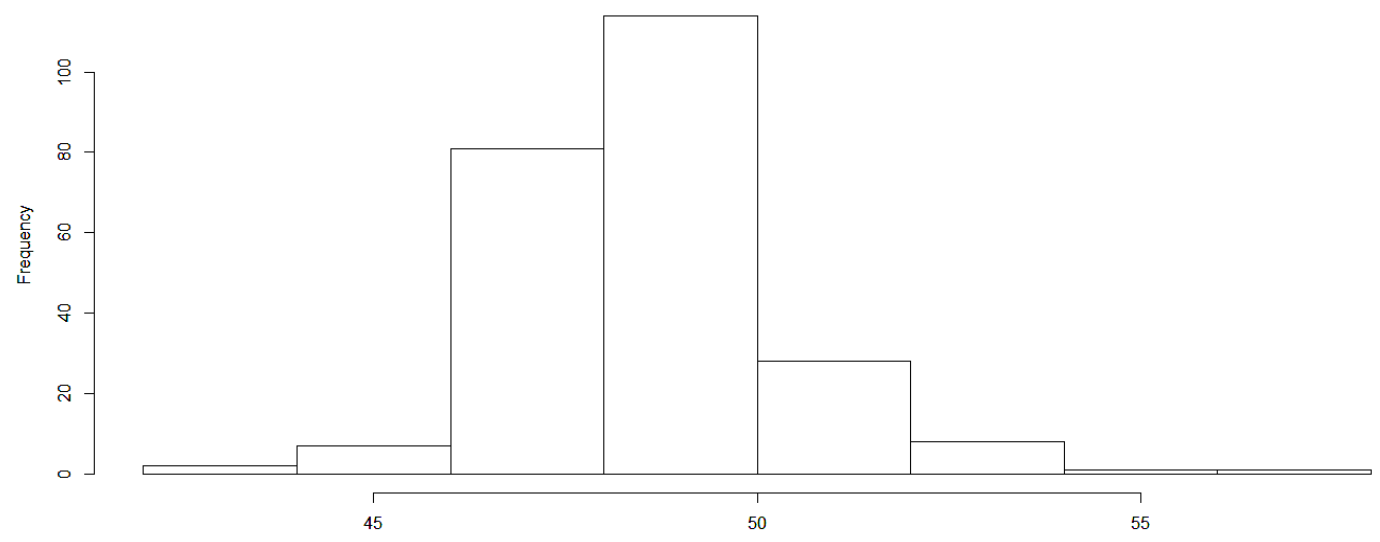

Fig. 2. Output power (in MW) distribution at the generating unit 2 of Asam-asam between 01/12/2016 and 14/12/2016. 
As reported in [4], the vacuum pressure at the main steam condenser has an increase of 20 percent. This may suggest that the main steam leaving the turbine still has a sufficiently high enthalpy (total energy), or the fouling growth inside the condenser is unusually rapid, or else. Any one of these may contribute to the above-mentioned decrease in the output. However, this study only considered variables observed on equipment and systems directly connected to the turbine. For examples, several devices like the deaerator (used to remove gas from liquid feed water) and the pulverizer (which supplies the coal powder) may perform processes whose variables fluctuate in values in a pattern consistent with that of output power.

\section{Modelling engineering systems}

Complex engineering systems involve a large number of variables. These variables carry with them information concerning the operation conditions of the systems. Consequently, analysis of these variables and the relationships between them is essential for explaining the behavior and the performance of the systems. These variables can be considered as random variables whose observed values are subject to random chances, and their analysis requires an approach that accommodates their probabilistic behaviors.

According to [5], especially under unstable conditions, engineering processes are better monitored using methods based on automatic process control. This approach, however, does not necessary provide opportunities for studying the root causes of the problems. In [6] it is clear that statistical process control does provide such opportunities. Unfortunately, the latter requires that the process be stabilized first before observations can result in any meaningful information about the behaviors.

A promising approach is based on modelling such behaviors. An approach centered on system assets in information modelling has been proposed by [7]. The use of statistical modelling techniques such as those with regression and multivariate models is required when the number of variables involved is large and they have to be simultaneously observed as multivariate variables. Several applications have been reported for monitoring and controlling engineering processes as in, e.g., $[8,9]$.

Generalized additive models (GAMs) can be used to fit engineering data using smoothing terms through addition. The general form is given, e.g., in [10] as follows:

$$
g\left(\mu_{i}\right)=\beta_{0}+\sum_{j=1}^{k} f_{j}\left(x_{i j}\right)
$$

where the link function $g\left(\mu_{i}\right)$, with $\mu_{i}$ being the expected value of the $i^{\text {th }}$ observation, is given as a linear combination of $k$ smoothing functions or smoothing terms $f_{j}\left(x_{i j}\right)$ and the intercept $\beta_{0}$. The function $g\left(\mu_{i}\right)$ is chosen to be associated with a certain exponential family of probability functions such as the normal family or the gamma family. For instance, the empirical distribution in Fig. 2 may be modelled using (1) where an $f_{j}$ would be a smoothing function of one of the other system variables. Computing packages are now available for this purpose in a statistical programming language like $\mathrm{R}$.

\section{Methods}

The modelling approach chosen in this study was based on generalized additive models. This allows relationships to be inferred between quantitative values in data in an additive fashion, thoroughly tested, and easily verified and understood. It also allows function smoothing to be performed on the relationship.

The object was chosen to be the power generating system of generating unit 2 at the Asam-asam steam power plant in South Kalimantan. Data were taken from the already available $\log$ sheets at the plant with permission, that is, from $01 / 12 / 2016$ to $14 / 12 / 2016$. The total number of variables observed was 144 . There were 151 observations for each of these variables. Part of a $\log$ sheet used at the plant is shown in Fig. 3.

This is a period of time that saw several processes involved in the generating system of that particular unit being in their unstable conditions. This means that the data represent those processes' states of instability. A general overhaul was carried out in the beginning of the following year.

The resulting matrix of data was then fed to the model as in (1) using an interface of the programming language R (available from http://cran.r-project.org). The $\mathrm{mgcv}$ package in $\mathrm{R}$ was used to fit the data.

\section{Data analysis and results}

Before applying model (1) it would be useful for the modelling process to reduce the number of system variables. This was done by screening for system variables having a sufficiently high coefficient of determination $\left(R^{2}\right)$ with output power. Such variables and their location in the cycle are given in Table 1.

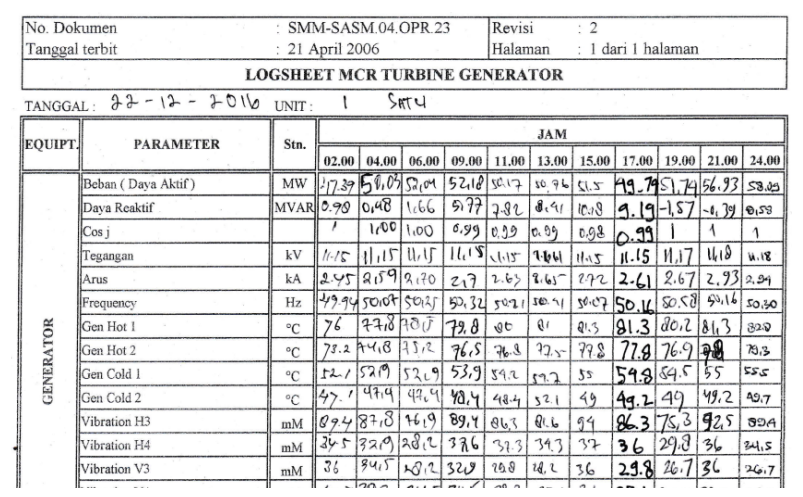

Fig. 3. Part of a log sheet used at the plant. 
Generated current is the electrical current generated in the generator. First stage pressure is the pressure of the first stage turbine. Condensate outlet pressure is the pressure of the condensed water leaving the low-pressure heater. Extracted steam pressure is the pressure of steam extracted from water by the deaerator. Feed water tank pressure is the pressure inside the feed water tank. Generated current and first stage pressure are directly related to output power at the generator. Hence, their relationship with output power is obvious. The other three variables, however, are not directly related to output power.

Table 1. Variables screened for the model.

\begin{tabular}{|l|l|c|}
\hline \multicolumn{1}{|c|}{ Variable } & \multicolumn{1}{c|}{ Location } & Symbol \\
\hline Generated current & Generator & $x_{1}$ \\
\hline First stage pressure & Turbine & $x_{2}$ \\
\hline $\begin{array}{l}\text { Condensate outlet } \\
\text { pressure }\end{array}$ & $\begin{array}{l}\text { Low-pressure } \\
\text { heater }\end{array}$ & $x_{3}$ \\
\hline Extracted steam pressure & Deaerator & $x_{4}$ \\
\hline Feed water tank pressure & Feed water tank & $x_{5}$ \\
\hline
\end{tabular}

Model fitting was performed using $\mathrm{R}$ programming language. The distribution family chosen for the expected value of output power is a gamma distribution. The shape of the histogram in Fig. 2 has motivated this choice. This requires the use of an inverse link function [10]. The model is given as follows:

$$
1 / \log \left(\mu_{i}\right)=\sum_{j=1}^{5} f_{j}\left(x_{i j}\right)
$$

In general, the five system variables can explain more than 83 percent of variation in output power value. The other 138 variables are responsible for less than 17 percent of the variation. The significance of the effect of the five variables can be seen from the resulting probability value ( $p$-Value) of their corresponding smoothing term. The smaller this value is, the more significant the effect will be. This is given in Table 2 .

Table 2. Significance of system variables.

\begin{tabular}{|l|c|}
\hline \multicolumn{1}{|c|}{ Variable } & p-Value \\
\hline Generated current & 0.001428 \\
\hline First stage pressure & 0.012185 \\
\hline Condensate outlet pressure & 0.000734 \\
\hline Extracted steam pressure & 0.001069 \\
\hline Feed water tank pressure & 0.000776 \\
\hline
\end{tabular}

The overall fit of the model can also be seen from its adjusted $R^{2}$ value $(0.805)$ and its generalized cross validation score $\left(3.26 \times 10^{-6}\right)$. A higher $R^{2}$ value and a smaller generalized cross validation score mean a better fit [10].
It is obvious that there is a strong relationship between generated current and the output power. However, it is quite surprising that variables condensate outlet pressure and feed water tank pressure contribute to variation in output power more significantly than the rest of the system variables. From an engineering viewpoint, the two variables are not directly related to output power at the generator, and this relationship might not be apparent from the measured values of those variables. The generalized additive model used for this purpose was based on a gamma distribution family. The link function is an inverse of a logarithmic function which makes the function more sensitive to changes in the two variables.

This result shows how it is possible discover empirical relationships between variables previously ignored or considered trivial. The model provides opportunities to study an engineering system even when it is at its unstable state.

Information from such a study can be very useful for operation and maintenance purposes. For instance, it might be recommended that the engineer in-charge of the operation look at the processes involving the corresponding variables. Although the model can also be used to predict the values of output power, its main purpose is to explain the variation of the variable.

\section{Conclusions and recommendations}

A steady decrease in output power of a steam power plant generating unit is a problem faced at the Asamasam power plant. It is important to explain this decrease. In doing so, it is necessary to be able to explain the effects of hundreds of variables on output power. The approach based on conventional thermodynamic analysis is not sufficient for this purpose since several assumptions have been violated by actual operation conditions.

The study has demonstrated how it is possible to model a complex engineering system, i.e. a power generating system using statistical modelling even when it is at its unstable state. The complexity comes from the fact that the number of variables involved is large and the empirical relationships between variables are not necessarily known.

A generalized additive model has been used to model the relationship between output power and five other variables. By fitting this model, it is discovered that condensate outlet pressure and feed water tank pressure contribute very significantly to changes in output power. It is recommended that this study be taken further to examine the implementation of the modelling results on the actual operation of the system. 
The author would like to thank Mr. Andi Y. Iriyanto from PT. PLN at the Asam-asam power plant for his assistance during the data collecting process, and students under his supervision at the Mechanical Eng. Dept., Lambung Mangkurat Univ. for preparing a digital version of the data.

\section{References}

1. Y. A. Çengel and M. A. Boles, Thermodynamics: An Engineering Approach $8^{\text {th }}$ Ed. (McGraw-Hill Education, New York, NY, 2015).

2. H. F. Martin, "Steam Turbines for Power Generation", in K. R. Rao (ed.), Energy and Power Generation Handbook: Established and Emerging Technologies (ASME Press, New York, NY, 2011).

3. C. Emmanouilidis and P. Pistofidis, "Wireless Condition Monitoring and Embedded Novelty Detection", in J. E. Amadi-Echendu, K. Brown, R. Willett, and J. Mathew (eds.), Definitions, Concepts and Scope of Engineering Asset Management (Springer-Verlag, London, 2010).

4. A. Y. Iriyanto, Analysis of Steam Turbine Performance at the Generating Unit 2 of the Asamasam Power Generating Plant (transl. unpublished Final Project Report) (Department of Mechanical Eng., Lambung Mangkurat University, 2017).
5. G. E. P. Box, D. E. Coleman, and R. V. Baxley, Jr. "A comparison of statistical process control and engineering process control" Journal of Quality Technology, 29 (1997): 128-130.

6. H. S. Gitlow, A. J. Oppenheim, R. Oppenheim, and D. M. Levine, Quality Management $3^{\text {rd }}$ Ed. (McGraw-Hill, New York, NY, 2005).

7. N. Fujisawa, S. Matsuo, Y. Yamamoto, and K. Nonaka, "Thermal power plant asset management with asset-centric data model", Fujitsu Sci. Tech. J. 50 (2014): 76-84.

8. J. F. MacGregor, H. Yu, S. G. Munoz, and J. FloresCerrilo, "Data-based latent variable methods for process analysis, monitoring and control", Computers and Chemical Eng. 29 (2005): 12171223.

9. L. Pan, D. Flynn, and M. Cregan, "Statistical model for power plant performance monitoring and analysis", Universities Power Eng. Conf. (2007): 121-126.

10. A. Agresti, Categorical Data Analysis $2^{\text {nd }}$ Ed. (John Wiley \& Sons, Inc., Hoboken, NJ, 2002). 